\title{
Greedy approximation and the multivariate Haar system
}

by

\author{
A. Kamont (Sopot) and V. N. Temlyakov (Columbia, SC)
}

\begin{abstract}
We study nonlinear $m$-term approximation in a Banach space with regard to a basis. It is known that in the case of a greedy basis (like the Haar basis $\mathcal{H}$ in $L_{p}([0,1])$, $1<p<\infty)$ a greedy type algorithm realizes nearly best $m$-term approximation for any individual function. In this paper we generalize this result in two directions. First, instead of a greedy algorithm we consider a weak greedy algorithm. Second, we study in detail unconditional nongreedy bases (like the multivariate Haar basis $\mathcal{H}^{d}=\mathcal{H} \times \ldots \times \mathcal{H}$ in $\left.L_{p}\left([0,1]^{d}\right), 1<p<\infty, p \neq 2\right)$. We prove some convergence results and also some results on convergence rate of weak type greedy algorithms. Our results are expressed in terms of properties of the basis with respect to a given weakness sequence.
\end{abstract}

1. Introduction. This paper deals with nonlinear $m$-term approximation with respect to a basis. Let $X$ be an infinite-dimensional separable Banach space with a norm $\|\cdot\|:=\|\cdot\|_{X}$ and let $\Psi:=\left\{\psi_{n}\right\}_{n=1}^{\infty}$ be a normalized basis for $X\left(\left\|\psi_{n}\right\|=1, n \in \mathbb{N}\right)$. All bases considered in this paper are assumed to be normalized. For a given $f \in X$ we define the best $m$-term approximation with regard to $\Psi$ as follows:

$$
\sigma_{m}(f, \Psi):=\sigma_{m}(f, \Psi)_{X}:=\inf _{b_{k}, \Lambda}\left\|f-\sum_{k \in \Lambda} b_{k} \psi_{k}\right\|_{X}
$$

where the inf is taken over coefficients $b_{k}$ and sets $\Lambda$ of indices with cardinality $\# \Lambda=m$. There is a natural algorithm of constructing an $m$-term approximant. For a given element $f \in X$ we consider the expansion

$$
f=\sum_{k=1}^{\infty} c_{k}(f, \Psi) \psi_{k} .
$$

We call a permutation $\varrho, \varrho(j)=k_{j}, j=1,2, \ldots$, of the positive integers

2000 Mathematics Subject Classification: 41A65, 41A46.

This research was supported by the National Science Foundation Grant DMS 0200187 and by ONR Grant N00014-91-J1343. It was done when A. Kamont was visiting Department of Mathematics, University of South Carolina in 2001. A. Kamont's visit was supported by DoD/DEPSCoR grant N00014-99-1-0547. 
decreasing and write $\varrho \in D(f)$ if

$$
\left|c_{k_{1}}(f, \Psi)\right| \geq\left|c_{k_{2}}(f, \Psi)\right| \geq \ldots
$$

In the case of strict inequalities here $D(f)$ consists of only one permutation. We define the $m$ th greedy approximant of $f$ with regard to the basis $\Psi$ corresponding to a permutation $\varrho \in D(f)$ by

$$
G_{m}(f, \Psi):=G_{m}^{X}(f, \Psi):=G_{m}(f, \Psi, \varrho):=\sum_{j=1}^{m} c_{k_{j}}(f, \Psi) \psi_{k_{j}} .
$$

It is a simple algorithm which describes a theoretical scheme (it is not computationally ready) for $m$-term approximation of an element $f$. This algorithm is known in the theory of nonlinear approximation under the name of Greedy Algorithm (see for instance [T2], [T3], [W]) and under the more specific name of Thresholding Greedy Algorithm (TGA) (see [T8], [DKKT]). We will use the latter name in this paper. The best we can achieve with the algorithm $G_{m}$ is

$$
\left\|f-G_{m}(f, \Psi, \varrho)\right\|_{X}=\sigma_{m}(f, \Psi)_{X}
$$

or a little weaker

$$
\left\|f-G_{m}(f, \Psi, \varrho)\right\|_{X} \leq G \sigma_{m}(f, \Psi)_{X}
$$

for all $f \in X$ with a constant $G=C(X, \Psi)$ independent of $f$ and $m$. The following concept of a greedy basis has been introduced in $[\mathrm{KT}]$.

Definition 1.1. We call a basis $\Psi$ a greedy basis if for every $f \in X$ there exists a permutation $\varrho \in D(f)$ such that

$$
\left\|f-G_{m}(f, \Psi, \varrho)\right\|_{X} \leq G \sigma_{m}(f, \Psi)_{X}
$$

with a constant independent of $f, m$.

The first result in this direction (see [T2]) was that the univariate Haar basis is a greedy basis. We recall the definition of the Haar basis. Let $\mathcal{H}:=$ $\left\{H_{k}\right\}_{k=1}^{\infty}$ be the Haar basis on $[0,1)$ normalized in $L_{2}(0,1): H_{1}=1$ on $[0,1)$ and for $k=2^{n}+l$, where $n=0,1, \ldots$ and $l=1,2, \ldots, 2^{n}$,

$$
H_{k}(x)= \begin{cases}2^{n / 2}, & x \in\left[(2 l-2) 2^{-n-1},(2 l-1) 2^{-n-1}\right), \\ -2^{n / 2}, & x \in\left[(2 l-1) 2^{-n-1}, 2 l 2^{-n-1}\right), \\ 0, & \text { otherwise. }\end{cases}
$$

We denote by $\mathcal{H}_{p}:=\left\{H_{k, p}\right\}_{k=1}^{\infty}$ the Haar basis $\mathcal{H}$ renormalized in $L_{p}(0,1)$.

The following weak type greedy algorithm was considered in [T2]. Let $t \in(0,1]$ be a fixed parameter. For a given basis $\Psi$ and a given $f \in X$ denote by $\Lambda_{m}(t)$ any set of $m$ indices such that

$$
\min _{k \in \Lambda_{m}(t)}\left|c_{k}(f, \Psi)\right| \geq t \max _{k \notin \Lambda_{m}(t)}\left|c_{k}(f, \Psi)\right|
$$


and define

$$
G_{m}^{X, t}(f, \Psi):=\sum_{k \in \Lambda_{m}(t)} c_{k}(f, \Psi) \psi_{k} .
$$

It was proved in [T2] that if $X=L_{p}, 1<p<\infty$, and $\Psi$ is the Haar system $\mathcal{H}$ then for any $f \in L_{p}$,

$$
\left\|f-G_{m}^{L_{p}, t}(f, \mathcal{H})\right\|_{L_{p}} \leq C(p, t) \sigma_{m}(f, \mathcal{H})_{L_{p}} .
$$

We note here that the proof of (1.5) from [T2] works for any greedy basis in place of the Haar system $\mathcal{H}$. Thus for any greedy basis $\Psi$ of a Banach space $X$ and any $t \in(0,1]$ we have, for each $f \in X$,

$$
\left\|f-G_{m}^{X, t}(f, \Psi)\right\|_{X} \leq C(p, t) \sigma_{m}(f, \Psi)_{X}
$$

This means that for greedy bases we have more flexibility in constructing nearly best $m$-term approximants.

Recently, in the theory of greedy algorithms with regard to redundant systems the Weak Greedy Algorithm with an arbitrary weakness sequence $\tau:=\left\{t_{k}\right\}_{k=1}^{\infty}$ has been studied (see [T7], [LTe], [T9]). In this paper we study its modification aimed at a further weakening of the restriction (1.4). We call this modification the Weak Thresholding Greedy Algorithm (WTGA). Let a weakness sequence $\tau:=\left\{t_{k}\right\}_{k=1}^{\infty}, t_{k} \in[0,1], k=1,2, \ldots$, be given. We define the WTGA by induction. We take an element $f \in X$ and at the first step we let

$$
\Lambda_{1}(\tau):=\left\{n_{1}\right\}, \quad G_{1}^{\tau}(f, \Psi):=c_{n_{1}} \psi_{n_{1}},
$$

with any $n_{1}$ satisfying

$$
\left|c_{n_{1}}\right| \geq t_{1} \max _{n}\left|c_{n}\right|
$$

where we write for brevity $c_{n}:=c_{n}(f, \Psi)$. Assume we have already defined

$$
G_{m-1}^{\tau}(f, \Psi):=G_{m-1}^{X, \tau}(f, \Psi):=\sum_{n \in \Lambda_{m-1}(\tau)} c_{n} \psi_{n}
$$

Then at the $m$ th step we define

$$
\Lambda_{m}(\tau):=\Lambda_{m-1}(\tau) \cup\left\{n_{m}\right\}, \quad G_{m}^{\tau}(f, \Psi):=G_{m}^{X, \tau}(f, \Psi):=\sum_{n \in \Lambda_{m}(\tau)} c_{n} \psi_{n},
$$

with any $n_{m} \notin \Lambda_{m-1}(\tau)$ satisfying

$$
\left|c_{n_{m}}\right| \geq t_{m} \max _{n \notin \Lambda_{m-1}(\tau)}\left|c_{n}\right| .
$$

Thus for an $f \in X$ the WTGA builds a rearrangement of a subsequence of the expansion (1.1). If $\Psi$ is an unconditional basis then we always have $G_{m}^{\tau}(f, \Psi) \rightarrow f^{*}$. It is clear that in this case $f^{*}=f$ if and only if the sequence $\left\{n_{k}\right\}_{k=1}^{\infty}$ contains the indices of all nonzero $c_{n}(f, \Psi)$. We say that 
the WTGA corresponding to $\Psi$ and $\tau$ is convergent (converges) if for any realization $G_{m}^{\tau}(f, \Psi)$ we have

$$
\left\|f-G_{m}^{\tau}(f, \Psi)\right\| \rightarrow 0 \quad \text { as } m \rightarrow \infty
$$

for all $f \in X$.

In Section 2 we prove the following three theorems on convergence of the WTGA. The first one deals with an arbitrary Banach space $X$ and any basis $\Psi$.

THEOREM 1. Let $X$ be a Banach space with a normalized basis $\Psi$. Let $\tau=\left\{t_{n}, n \geq 1\right\}$ be a weakness sequence. The following condition (D) is a necessary condition for the WTGA corresponding to $\Psi$ and $\tau$ to be convergent.

(D) For each subsequence $\left\{n_{k}, k \geq 1\right\}$ of different indices, the series $\sum_{k=1}^{\infty} t_{k} \psi_{n_{k}}$ diverges in $X$.

If the basis $\Psi$ is unconditional, then (D) is also sufficient condition for the convergence of the WTGA corresponding to $\Psi$ and $\tau$.

In the case $X=L_{p}\left([0,1]^{d}\right)$ we can derive from Theorem 1 a more specific condition in terms of $\tau$.

TheOrem 2. Let $2 \leq p<\infty, d \geq 1$ and let $\Psi$ be a normalized unconditional basis in $L_{p}\left([0,1]^{d}\right)$. Let $\tau=\left\{t_{n}, n \geq 1\right\}$ be a weakness sequence. Then the WTGA corresponding to $\Psi$ and $\tau$ converges if and only if $\tau \notin l_{p}$.

We do not have such a simple criterion in terms of $\tau$ for $X=L_{p}\left([0,1]^{d}\right)$, $1<p<2$, and arbitrary unconditional basis $\Psi$. In this case we have the following result for the multivariate Haar basis $\mathcal{H}_{p}^{d}$ defined as the tensor product of the univariate Haar bases: $\mathcal{H}_{p}^{d}:=\mathcal{H}_{p} \times \ldots \times \mathcal{H}_{p}$. To formulate the result, we introduce the following notation. For a sequence $\left\{t_{k}, k \geq 1\right\}$ of nonnegative numbers such that $\lim _{k \rightarrow \infty} t_{k}=0,\left\{t_{k}^{*}, k \geq 1\right\}$ is a nonincreasing rearrangement of the subsequence $\left\{t_{n_{k}}, k \geq 1\right\}$ consisting of the positive elements of $\left\{t_{k}, k \geq 1\right\}$.

THEOREM 3. Let $d \geq 1$ and $1<p<2$. The WTGA corresponding to $\mathcal{H}_{p}^{d}$ and a weakness sequence $\tau$ converges in $L_{p}\left([0,1]^{d}\right)$ if and only if one of the following conditions is satisfied:

(i) The sequence $\tau=\left\{t_{k}\right\}$ does not converge to 0 .

(ii) $\lim _{k \rightarrow \infty} t_{k}=0$ and

$$
\sum_{k=2}^{\infty}\left(t_{k}^{*}\right)^{2}\left(k(\log k)^{1-d}\right)^{2 / p-1}=\infty .
$$

Along with convergence of the WTGA we study efficiency of approximation by $G_{m}^{\tau}(\cdot, \Psi)$. We compare accuracy of the WTGA with best $m$-term 
approximation. In the case of a greedy basis and $\tau=\{t\}, t \in(0,1]$, the relation (1.6) shows that $G_{m}^{\tau}(\cdot, \Psi)$ realizes nearly best $m$-term approximation. There are two natural ways of adapting (1.6) to the case of nongreedy bases or general weakness sequences. The first way (see [T5], [T3], [W], [Os]) is to write (1.6) in the form

$$
\left\|f-G_{m}^{\tau}(f, \Psi)\right\| \leq C(m, \tau, \Psi) \sigma_{m}(f, \Psi)
$$

and look for the best (in the sense of order) constant $C(m, \tau, \Psi)$.

We now formulate the corresponding results. For a basis $\Psi$ we define the fundamental function

$$
\varphi(m):=\sup _{\# A \leq m}\left\|\sum_{k \in A} \psi_{k}\right\|
$$

We also need the functions

$$
\varphi^{\mathrm{s}}(m):=\sup _{\# A=m}\left\|\sum_{k \in A} \psi_{k}\right\|, \quad \varphi^{\mathrm{i}}(m):=\inf _{\# A=m}\left\|\sum_{k \in A} \psi_{k}\right\| .
$$

It is clear that

$$
\varphi(m)=\sup _{n \leq m} \varphi^{\mathrm{s}}(n)
$$

We now introduce some characteristics of a basis with respect to a weakness sequence $\tau$. For a subset $V \subseteq[1, m]$ of integers we define

$$
\phi(\tau, m, V):=\inf _{\left\{k_{i}\right\}}\left\|\sum_{i \in V} t_{i} \psi_{k_{i}}\right\|
$$

where the inf is taken over all sets $\left\{k_{i}\right\}$ of different indices. For two integers $1 \leq n \leq m$ we define

$$
\phi(\tau, m, n):=\inf _{\# V=n} \phi(\tau, m, V),
$$

and finally

$$
\mu(\tau, m):=\sup _{n \leq m} \frac{\varphi^{\mathrm{s}}(n)}{\phi(\tau, m, n)} .
$$

We have the following result.

TheOREM 4. Let $\Psi$ be a normalized unconditional basis for $X$. Then

$$
\left\|f-G_{m}^{\tau}(f, \Psi)\right\| \leq C(\Psi) \mu(\tau, m) \sigma_{m}(f, \Psi) .
$$

In the case $\tau=\{1\}$ Theorem 4 is known. The first result in this direction was obtained for the multivariate Haar basis $\mathcal{H}_{p}^{d}$ (see [T3]). Then it was generalized in $[\mathrm{W}]$ to other bases, in particular, to normalized unconditional bases. Moreover, it has been proved in [W] that $\mu_{m}(\{1\}, m)$ is an optimal extra factor in the above inequality for $\tau=\{1\}$.

In Theorem 4 we compare efficiency of $G_{m}^{\tau}(\cdot, \Psi)$ with $\sigma_{m}(\cdot, \Psi)$. It is known in approximation theory that sometimes it is convenient to compare 
efficiency of an approximating operator which is characterized by $m$ parameters with best possible approximation corresponding to a smaller number of parameters $n \leq m$. We use this idea in approximation by the WTGA. In this paper we study a setting when we write (1.6) in the form

$$
\left\|f-G_{v_{m}}^{\tau}(f, \Psi)\right\| \leq C(\Psi) \sigma_{m}(f, \Psi)
$$

and look for the best (in the sense of order) sequence $\left\{v_{m}\right\}$ that is determined by the weakness sequence $\tau$ and the basis $\Psi$. We need some more notation. Define

$$
\phi(\tau, N):=\phi(\tau, N,[1, N])=\inf _{k_{1}, \ldots, k_{N}}\left\|\sum_{j=1}^{N} t_{j} \psi_{k_{j}}\right\| .
$$

Assume that $\phi(\tau, N) \rightarrow \infty$ as $N \rightarrow \infty$ and denote by $v_{m}$ the smallest $N$ satisfying

$$
\phi(\tau, N) \geq 2 \varphi(m) .
$$

We have the following result in this case.

TheOREM 5. For any normalized unconditional basis $\Psi$ we have

$$
\left\|f-G_{v_{m}}^{\tau}(f, \Psi)\right\| \leq C(\Psi) \sigma_{m}(f, \Psi) .
$$

It is interesting to compare this result with some recent results from [DKKT]. It has been established in [DKKT] that the inequalities

$$
\left\|f-G_{\lambda m}(f, \Psi)\right\| \leq C(\Psi, \lambda) \sigma_{m}(f, \Psi)
$$

with fixed $\lambda>1$ are characteristic for a special class of bases. We describe this class now. Let us say that a basis $\Psi$ is almost greedy if there is a constant $C$ so that for any $f \in X$,

$$
\left\|f-G_{m}(f, \Psi)\right\| \leq C \inf _{\Lambda, \# \Lambda=m}\left\|f-\sum_{k \in \Lambda} c_{k}(f, \Psi) \psi_{k}\right\| .
$$

It is clear that each greedy basis is almost greedy. It has been proved in [DKKT] that if (1.8) holds for some $\lambda>1$ and all $f \in X$ then $\Psi$ is almost greedy. It has also been proved in [DKKT] that (1.8) holds for any $\lambda>1$ and all $f \in X$ provided $\Psi$ is almost greedy.

When the results of this paper were completed (see survey [KTe]) we learned from P. Wojtaszczyk that he had also obtained results in the style of Theorem 5 in the case $\tau=\{1\}$ (see Theorem 4 of [Wo]).

In Section 4 we discuss the greedy properties of subsequences of the Haar basis $\mathcal{H}_{p}^{d}:=\mathcal{H}_{p} \times \ldots \times \mathcal{H}_{p}$ that is the tensor product of the univariate Haar bases $\mathcal{H}$. It is known (see [T2] and [T3]) that $\mathcal{H}_{p}$ is a greedy basis for $L_{p}([0,1]), 1<p<\infty$, and $\mathcal{H}_{p}^{d}$ is a greedy basis for $L_{p}\left([0,1]^{d}\right), d \geq 2$, only for $p=2$. Let $\mathcal{M}$ be a subset of the set of indices $\mathbf{n} \in \mathbb{Z}_{+}^{d}$. We define

$$
\mathcal{H}_{p}^{d}[\mathcal{M}]:=\left\{H_{\mathbf{n}, p}, \mathbf{n} \in \mathcal{M}\right\},
$$




$$
L_{p}[\mathcal{M}]:=\left\{f \in L_{p}\left([0,1]^{d}\right):\left\langle f, H_{\mathbf{n}}\right\rangle=0, \mathbf{n} \notin \mathcal{M}\right\}=\overline{\operatorname{span}}\left\{\mathcal{H}_{p}^{d}[\mathcal{M}]\right\},
$$

where the closure is taken in $L_{p}\left([0,1]^{d}\right)$.

We introduce some more notation. Let us define the decomposition of $\mathcal{H}_{p}^{d}$ into dyadic blocks. First, define

$$
U_{0}:=\{1,2\}, \quad U_{s}:=\left\{n \in \mathbb{N}: 2^{s}+1 \leq n \leq 2^{s+1}\right\} \quad \text { for } s \geq 1 .
$$

For $\mathbf{s}=\left(s_{1}, \ldots, s_{d}\right)$ we set

$$
U_{\mathbf{s}}:=\left\{\mathbf{n}=\left(n_{1}, \ldots, n_{d}\right): n_{i} \in U_{s_{i}} \text { for } i=1, \ldots, d\right\} .
$$

We note that for each $\mathbf{s}$ the supports of the functions $\left\{H_{\mathbf{n}, p}, \mathbf{n} \in U_{\mathbf{s}}\right\}$ have the same shape and measure $2^{-|\mathbf{s}|}$, where $|\mathbf{s}|=s_{1}+\ldots+s_{d}$. Moreover, if $\mathbf{s}=\left(s_{1}, \ldots, s_{d}\right)$ with $s_{i} \neq 0$ for all $1 \leq i \leq d$, then $\# U_{\mathbf{s}}=2^{|\mathbf{s}|}$ and the supports of the functions $\left\{H_{\mathbf{n}, p}, \mathbf{n} \in U_{\mathbf{s}}\right\}$ are disjoint. For general $\mathbf{s}$ we have $2^{|\mathbf{s}|} \leq \# U_{\mathbf{s}} \leq 2^{|\mathbf{s}|+d}$, and at most $2^{d}$ different functions from $\left\{H_{\mathbf{n}, p}, \mathbf{n} \in U_{\mathbf{s}}\right\}$ have the same support.

For a positive constant $K$ we define two classes of subsequences $\mathcal{M}$ :

$$
\begin{aligned}
\mathbf{R}(K) & :=\left\{\mathcal{M}: \forall n \#\left\{\mathbf{s}: \mathcal{M} \cap U_{\mathbf{s}} \neq \emptyset,|\mathbf{s}|=n\right\} \leq K\right\}, \\
\mathbf{J}(K) & :=\left\{\mathcal{M}: \forall \mathbf{s} \#\left(\mathcal{M} \cap U_{\mathbf{s}}\right) \leq K\right\} .
\end{aligned}
$$

Denote by $\mathbf{G}(d)$ the set of all subsequences $\mathcal{M}$ representable in the form $\mathcal{M}=\mathcal{M}_{1} \cup \mathcal{M}_{2}$, where $\mathcal{M}_{1} \in \mathbf{R}\left(K_{1}\right)$ and $\mathcal{M}_{2} \in \mathbf{J}\left(K_{2}\right)$ with some constants $K_{1}, K_{2}$.

Theorem 6. Let $\mathcal{M} \in \mathbf{G}(d)$. Then $\mathcal{H}_{p}^{d}[\mathcal{M}]$ is a greedy basis for $L_{p}[\mathcal{M}]$, $1<p<\infty$.

It is clear that the condition $\mathcal{M} \in \mathbf{G}(d)$ is not necessary for $\mathcal{H}_{p}^{d}[\mathcal{M}]$ to be a greedy basis for $L_{p}[\mathcal{M}]$. Indeed, we can find a sequence $\mathcal{M} \notin \mathbf{G}(d)$ with disjoint supports of $H_{\mathbf{n}}, \mathbf{n} \in \mathcal{M}$. However, we will show in Section 4 that Theorem 6 is sharp in a certain sense.

In Section 5 we present results on relations between $\left\{\sigma_{m}\left(f, \mathcal{H}^{d}\right)_{p}\right\}$ and $\left\{c_{\mathbf{n}}\left(f, \mathcal{H}_{p}^{d}\right)\right\}$. We give some embedding theorems in terms of the Lorentz spaces and their slight modifications.

Let us agree to denote by $C$ various positive absolute constants and by $C$ with arguments or indices $\left(C(q, p), C_{r}\right.$ and so on) positive numbers which depend on the arguments indicated. For two nonnegative sequences $a=\left\{a_{n}\right\}_{n=1}^{\infty}$ and $b=\left\{b_{n}\right\}_{n=1}^{\infty}$ the relation (order inequality) $a_{n} \ll b_{n}$ means that there is a number $C(a, b)$ such that $a_{n} \leq C(a, b) b_{n}$ for all $n$; and the relation $a_{n} \asymp b_{n}$ means that $a_{n} \ll b_{n}$ and $b_{n} \ll a_{n}$.

Acknowledgements. We would like to thank Professor P. Wojtaszczyk and Professors S. J. Dilworth, N. J. Kalton and D. Kutzarova for letting us know early versions of their papers [Wo] and [DKK]. 
2. The convergence results. In this section we will prove and discuss Theorems 1-3.

Proof of Theorem 1. We begin with the necessity part. Our proof is by contradiction. Suppose that $\sum_{k=1}^{\infty} t_{k} \psi_{n_{k}}$ converges in $X$ for some sequence $\left\{n_{k}, k \geq 1\right\}$ of different indices.

First, we consider a special case. Let $\left\{n_{k}, k \geq 1\right\}$ be a sequence of different indices such that $\sum_{k=1}^{\infty} t_{k} \psi_{n_{k}}$ converges in $X$ and there is a $\nu \in \mathbb{N}$ such that $n_{k} \neq \nu$ for all $k \in \mathbb{N}$. Take

$$
f=\psi_{\nu}+\sum_{k=1}^{\infty} t_{k} \psi_{n_{k}}
$$

Then we can take the following realization of the WTGA:

$$
G_{m}^{\tau}(f, \Psi)=\sum_{k=1}^{m} t_{k} \psi_{n_{k}} .
$$

Thus

$$
f-G_{m}^{\tau}(f, \Psi)=\psi_{\nu}+\sum_{k=m+1}^{\infty} t_{k} \psi_{n_{k}}
$$

and $\left\|f-G_{m}^{\tau}(f, \Psi)\right\| \nrightarrow 0$. Consequently, the WTGA corresponding to $\Psi$ and $\tau$ is not convergent.

We now reduce the general case to the above special case. Let $\left\{n_{k}, k \geq 1\right\}$ be a sequence of different indices such that $\sum_{k=1}^{\infty} t_{k} \psi_{n_{k}}$ converges in $X$. This implies that $\lim _{k \rightarrow \infty} t_{k}=0$, so there is a subsequence $\left\{k_{l}, l \geq 1\right\}$ with $k_{1}=1$ such that

$$
\sum_{l=1}^{\infty} t_{k_{l}}<\infty
$$

Clearly, then both

$$
\sum_{l=1}^{\infty} t_{k_{l}} \psi_{n_{k_{l}}} \text { and } \sum_{l=1}^{\infty} t_{k_{l}} \psi_{n_{k_{l+1}}}
$$

converge in $X$, and

$$
\sum_{k=1}^{\infty} t_{k} \psi_{n_{k}}-\sum_{l=1}^{\infty} t_{k_{l}} \psi_{n_{k_{l}}}+\sum_{l=1}^{\infty} t_{k_{l}} \psi_{n_{k_{l+1}}}=\sum_{k=1}^{\infty} t_{k} \psi_{s_{k}}
$$

where

$$
s_{k}= \begin{cases}n_{k} & \text { if } k \neq k_{l} \text { for all } l \geq 1, \\ n_{k_{l+1}} & \text { if } k=k_{l} \text { for some } l \geq 1 .\end{cases}
$$

Note that $\left\{s_{k}, k \geq 1\right\}$ is a sequence of different indices such that $s_{k} \neq n_{1}$ for all $k \geq 1$. Therefore we are in the special case considered above. This completes the proof of the necessity part. 
We now proceed to the sufficiency part. Our proof is again by contradiction. Assume that $\Psi$ is an unconditional basis. Suppose that $f \in X$ is such that

$$
G_{m}^{\tau}(f, \Psi) \nrightarrow f
$$

in $X$. By definition,

$$
G_{m}^{\tau}(f, \Psi)=\sum_{k=1}^{m} c_{n_{k}} \psi_{n_{k}}
$$

where

$$
\left|c_{n_{1}}\right| \geq t_{1} \sup _{n \in \mathbb{N}}\left|c_{n}\right|, \quad\left|c_{n_{k}}\right| \geq t_{k} \sup _{n \neq n_{1}, \ldots, n_{k-1}}\left|c_{n}\right| \quad \text { for } k \geq 2 .
$$

As $G_{m}^{\tau}(f, \Psi) \nrightarrow f$ and the basis $\Psi$ is unconditional, there is $\mu \in \mathbb{N}$ with $c_{\mu} \neq 0$ such that $n_{k} \neq \mu$ for all $k \in \mathbb{N}$. Hence (2.1) implies that $t_{k} \leq$ $\left|c_{n_{k}}\right| /\left|c_{\mu}\right|$. Since the basis $\Psi$ is unconditional, it follows that the series $\sum_{k=1}^{\infty} t_{k} \psi_{n_{k}}$ converges in $X$. Theorem 1 is now proved.

REMARK 2.1. In the case of conditional bases, the condition (D) is not sufficient for convergence of the corresponding algorithm. For example, it is clear that for TGA $(\tau=\{1\})$ this condition is always satisfied. However, the TGA may not converge for some bases. For instance, it was proved in [T5] (see also $[\mathrm{CF}]$ for $1 \leq p<2$ ) that the TGA may diverge in $L_{p}, p \neq 2$, for the trigonometric system.

Let us note that convergence of TGA can be used as a defining condition for a class of bases called quasi-greedy bases (see $[\mathrm{KT}]$, [W]; more precisely, the definition of a quasi-greedy basis in $[\mathrm{KT}]$ is different, and in $[\mathrm{W}]$ the equivalence of this definition and convergence of TGA is proved). It is clear that any unconditional basis is a quasi-greedy basis. It is known (see [KT]) that there is a quasi-greedy basis that is not an unconditional basis. For more examples of conditional quasi-greedy bases see [W], [DM]. We also remark that the question of existence of quasi-greedy bases in Banach spaces, in a very general setting, has been treated in the recent paper [DKK].

We will prove one technical result that we will need later on. Let $\mathcal{M}=$ $\left\{m_{k}, k \geq 1\right\}$ be a sequence of different indices, and let $\tau=\left\{t_{k}, k \geq 1\right\}$ be a weakness sequence. Consider a new weakness sequence $\tau(\mathcal{M})=\left\{\eta_{n}, n \geq 1\right\}$, where

$$
\eta_{n}= \begin{cases}t_{k} & \text { when } n=m_{k} \text { for } k \geq 1, \\ 0 & \text { otherwise }\end{cases}
$$

We have the following corollary of Theorem 1 .

Proposition 2.1. Let $\Psi$ be a normalized unconditional basis in a Banach space $X$. Then the WTGA corresponding to $\Psi$ and $\tau(\mathcal{M})$ is convergent if and only if the WTGA corresponding to $\Psi$ and $\tau$ is convergent. 
Proof. It is clear that if $\tau(\mathcal{M})$ does not satisfy the necessary and sufficient condition (D) from Theorem 1, then neither does $\tau$. Thus if the WTGA diverges for $\tau(\mathcal{M})$ it diverges for $\tau$. We now prove that if $\tau$ does not satisfy (D) then $\tau(\mathcal{M})$ also does not satisfy (D). Assume that $\sum_{k=1}^{\infty} t_{k} \psi_{n_{k}}$ converges. Then $t_{k} \rightarrow 0$ and we let $\mathcal{K}:=\left\{k_{j}\right\}_{j=1}^{\infty}$ be an infinite set such that

$$
\sum_{j=1}^{\infty} t_{k_{j}}<\infty
$$

Let $\mathcal{L}:=\mathbb{N} \backslash \mathcal{K}$ and note that the series $\sum_{k \in \mathcal{L}} t_{k} \psi_{n_{k}}$ also converges.

We now assign to each $\eta_{m_{k}}=t_{k}, k \in \mathcal{L}$, from the sequence $\tau(\mathcal{M})$ a basic function $\psi_{n_{k}}$. We split the infinite set $\mathcal{K}$ into a union of two infinite sets $\mathcal{K}_{1}$ and $\mathcal{K}_{2}$. Then we set up a one-to-one correspondence $k \leftrightarrow k^{\prime}$ between $\mathcal{K}$ and $\mathcal{K}_{1}$ and assign to each $\eta_{m_{k}}=t_{k}, k \in \mathcal{K}$, a basic function $\psi_{n_{k^{\prime}}}$; to different $\eta_{l}=0$ we assign different basic functions $\psi_{s}$ with $s \in \bigcup_{k \in \mathcal{K}_{2}}\left\{n_{k}\right\}$. Then the corresponding sum from the condition (D) for $\tau(\mathcal{M})$ will be

$$
\sum_{k \in \mathcal{L}} t_{k} \psi_{n_{k}}+\sum_{k \in \mathcal{K}} t_{k} \psi_{n_{k^{\prime}}}
$$

This series converges and therefore $\tau(\mathcal{M})$ does not satisfy (D). By Theorem 1 we conclude that the WTGA corresponding to $\tau(\mathcal{M})$ diverges. This completes the proof of Proposition 2.1.

Proof of Theorem 2. Since $\Psi$ is a normalized unconditional basis in $L_{p}\left([0,1]^{d}\right)$ with $p \geq 2$, for any set $\left\{n_{k}\right\}$ of different indices and $N \in \mathbb{N}$ we have

$$
\left\|\sum_{k=1}^{N} t_{k} \psi_{n_{k}}\right\|_{p} \geq C\left(\int_{[0,1]^{d}}\left(\sum_{k=1}^{N}\left|t_{k}\right|^{2}\left|\psi_{n_{k}}(x)\right|^{2}\right)^{p / 2} d x\right)^{1 / p} \geq C\left(\sum_{k=1}^{N}\left|t_{k}\right|^{p}\right)^{1 / p} .
$$

Therefore, by the sufficiency part of Theorem 1 the WTGA with a weakness sequence $\tau$ converges if $\tau \notin l_{p}$.

Assume now that $\tau \in l_{p}$. Then it is known (see [KP, Theorem 4]) that an unconditional basis $\Psi=\left\{\psi_{n}\right\}_{n=1}^{\infty}$ of $L_{p}\left([0,1]^{d}\right), 1<p<\infty$, contains a subsequence $\left\{\psi_{n_{k}}\right\}_{k=1}^{\infty}$ such that each series $\sum_{k=1}^{\infty} a_{k} \psi_{n_{k}}$ converges provided $\left\{a_{k}\right\}_{k=1}^{\infty} \in l_{p}$. Specifying $a_{k}=t_{k}$ and applying Theorem 1 we conclude that the WTGA with the weakness sequence $\tau$ does not converge. This completes the proof of Theorem 2 .

The case of $L_{p}\left([0,1]^{d}\right)$ with $1<p<2$ is different-the condition on the weakness sequence $\tau$ depends now on a particular unconditional basis $\Psi$. Let $\Psi$ be a normalized unconditional basis in $L_{p}\left([0,1]^{d}\right)$ with $1<p<2$. 
Then for any coefficients $\left\{a_{n}\right\}$ and $N \in \mathbb{N}$ we have

$$
C_{1}\left(\sum_{n=1}^{N}\left|a_{n}\right|^{2}\right)^{1 / 2} \leq\left\|\sum_{n=1}^{N} a_{n} \psi_{n}\right\|_{p} \leq C_{2}\left(\sum_{n=1}^{N}\left|a_{n}\right|^{p}\right)^{1 / p} .
$$

Thus by Theorem 1 if a weakness sequence $\tau \in l_{p}$, then the WTGA corresponding to $\Psi$ and $\tau$ is not convergent in $L_{p}\left([0,1]^{d}\right)$. Also, if $\tau \notin l_{2}$ then the WTGA corresponding to $\Psi$ and $\tau$ is convergent in $L_{p}\left([0,1]^{d}\right)$. In addition, as $L_{p} \oplus l_{2}$ is isomorphic to $L_{p}$, there is an unconditional basis $\Psi$ in $L_{p}\left([0,1]^{d}\right)$ $(1<p<2)$ for which the condition $\tau \notin l_{2}$ is also a necessary condition for the WTGA corresponding to $\Psi$ and $\tau$ to be convergent.

Let us consider in detail the case of the Haar system $\mathcal{H}_{p}^{d}$.

Proof of Theorem 3. If $\tau=\left\{t_{k}, k \geq 1\right\}$ is a sequence of nonnegative numbers which does not converge to 0 , then $\lim _{\sup _{k \rightarrow \infty}} t_{k}>0$, and convergence of the WTGA corresponding to $\mathcal{H}_{p}^{d}$ and $\tau$ is an immediate consequence of Theorem 1.

It remains to consider sequences $\tau$ such that $\lim _{k \rightarrow \infty} t_{k}=0$. By Proposition 2.1, it is sufficient to consider a sequence $\tau$ with $t_{k} \neq 0$. Set

$$
q_{m}:=\#\left\{\mathbf{n}:\left|\operatorname{supp} H_{\mathbf{n}}\right|=2^{-m}\right\}, \quad \nu_{0}=0, \quad \nu_{m}=\sum_{j=0}^{m-1} q_{j} \quad \text { for } m \geq 1 .
$$

Note that $q_{m} \asymp m^{d-1} 2^{m} \asymp \nu_{m}$ and $\log q_{m} \asymp m \asymp \log \nu_{m}$ for $m \geq 1$. As the sequence $\left\{t_{k}^{*}, k \geq 0\right\}$ is nonincreasing, for $\nu_{m} \leq k \leq \nu_{m+1}$ we have

$$
C_{1}\left(t_{\nu_{m+1}}^{*}\right)^{2} 2^{m(2 / p-1)} \leq\left(t_{k}^{*}\right)^{2}\left(k(\log k)^{1-d}\right)^{2 / p-1} \leq C_{2}\left(t_{\nu_{m}}^{*}\right)^{2} 2^{m(2 / p-1)},
$$

which implies that

$$
\sum_{k=1}^{\infty}\left(t_{k}^{*}\right)^{2}\left(k(\log k)^{1-d}\right)^{2 / p-1}<\infty \Leftrightarrow \sum_{m=1}^{\infty}\left(t_{\nu_{m+1}}^{*}\right)^{2} m^{d-1} 2^{2 m / p}<\infty .
$$

Let us recall (see Lemma 3.1 below for more detail) that for any $N$ different indices $\mathbf{n}_{1}, \ldots, \mathbf{n}_{N}$,

$$
\left\|\sum_{i=1}^{N} H_{\mathbf{n}_{i}, p}\right\|_{p} \geq C(p, d)(\log N)^{(d-1)(1 / 2-1 / p)} N^{1 / p}, \quad 1<p \leq 2 .
$$

For any sequence $\left\{\mathbf{n}_{k}, k \geq 1\right\}$ of different indices,

$$
\left\|\sum_{k=1}^{\infty} t_{k} H_{\mathbf{n}_{k}, p}\right\|_{p}=\left\|\sum_{k=1}^{\infty} t_{k}^{*} H_{\mathbf{n}_{k}^{*}, p}\right\|_{p} \geq C(p, d)\left(\sum_{m=0}^{\infty}\left\|\sum_{k=\nu_{m}+1}^{\nu_{m+1}} t_{k}^{*} H_{\mathbf{n}_{k}^{*}, p}\right\|_{p}^{2}\right)^{1 / 2},
$$

where $\mathbf{n}_{k}^{*}$ is such that $t_{k}^{*}=t_{k^{*}}$ and $\mathbf{n}_{k}^{*}=\mathbf{n}_{k^{*}}$. By (2.3) we continue the 
estimate:

$$
\begin{aligned}
& \geq C(p, d)\left(\sum_{m=0}^{\infty}\left(t_{\nu_{m+1}}^{*}\left(\log q_{m}\right)^{(d-1)(1 / 2-1 / p)} q_{m}^{1 / p}\right)^{2}\right)^{1 / 2} \\
& \geq C(p, d)\left(\sum_{m=0}^{\infty}\left(t_{\nu_{m+1}}^{*}\right)^{2}(m+1)^{d-1} 2^{2 m / p}\right)^{1 / 2} .
\end{aligned}
$$

Thus, it follows from (2.2) and Theorem 1 that if a weakness sequence $\tau$ satisfies the condition (1.7), then the WTGA corresponding to $\mathcal{H}_{p}^{d}$ and $\tau$ converges.

Suppose now that

$$
\sum_{k=1}^{\infty}\left(t_{k}^{*}\right)^{2}\left(k(\log k)^{1-d}\right)^{2 / p-1}<\infty .
$$

Take a sequence $\left\{\mathbf{n}_{k}, k \geq 1\right\}$ of different indices satisfying

$$
\left|\operatorname{supp} H_{\mathbf{n}_{k}, p}\right|=2^{-m} \quad \text { for } \nu_{m}+1 \leq k \leq \nu_{m+1},
$$

i.e. we order the functions $H_{\mathbf{n}, p}$ according to the measure of their supports (more precisely, the sequence $\left|\operatorname{supp} H_{\mathbf{n}_{k}, p}\right|$ is nonincreasing). Note that with this ordering we have $\sum_{k=\nu_{m}+1}^{\nu_{m+1}}\left|H_{\mathbf{n}_{k}, p}(x)\right|^{2} \asymp(m+1)^{d-1} 2^{2 m / p}$ for each $x \in[0,1]^{d}$. Then, using unconditionality of $\mathcal{H}_{p}^{d}$, we obtain

$$
\begin{aligned}
\left\|\sum_{k=1}^{\infty} t_{k}^{*} H_{\mathbf{n}_{k}, p}\right\|_{p}^{p} & \leq C(p, d) \int_{[0,1]^{d}}\left(\sum_{k=1}^{\infty}\left|t_{k}^{*} H_{\mathbf{n}_{k}, p}(x)\right|^{2}\right)^{p / 2} d x \\
& \leq C(p, d) \int_{[0,1]^{d}}\left(\sum_{m=1}^{\infty}\left(t_{\nu_{m}+1}^{*}\right)^{2} \sum_{k=\nu_{m}+1}^{\nu_{m+1}}\left|H_{\mathbf{n}_{k}, p}(x)\right|^{2}\right)^{p / 2} d x \\
& \leq C(p, d)\left(\sum_{m=0}^{\infty}\left(t_{\nu_{m}+1}^{*}\right)^{2}(m+1)^{d-1} 2^{2 m / p}\right)^{p / 2} .
\end{aligned}
$$

The above inequality combined with Theorem 1 and (2.2) implies that for $\tau$ satisfying (2.4) the corresponding WTGA is not convergent.

3. Proof of Theorems 4 and 5. This proof uses an idea from [T2] (see also $[\mathrm{KT}]$ ). The following proposition is a well known fact about unconditional bases (see [LT, vol. I, p. 19]).

Proposition 3.1. Let $\Psi$ be an unconditional basis for $X$. Then for every choice of bounded scalars $\left\{\lambda_{k}\right\}_{k=1}^{\infty}$, we have

$$
\left\|\sum_{k=1}^{\infty} \lambda_{k} a_{k} \psi_{k}\right\| \leq K \sup _{k}\left|\lambda_{k}\right|\left\|\sum_{k=1}^{\infty} a_{k} \psi_{k}\right\| .
$$


Take any $\varepsilon>0$ and find

$$
p_{m}(f):=\sum_{k \in P} b_{k} \psi_{k}
$$

such that $\# P=m$ and

$$
\left\|f-p_{m}(f)\right\| \leq \sigma_{m}(f, \Psi)+\varepsilon .
$$

For any finite set $\Lambda$ of indices we denote by $S_{\Lambda}$ the projector

$$
S_{\Lambda}(f):=\sum_{k \in \Lambda} c_{k}(f, \Psi) \psi_{k} .
$$

Proposition 3.1 implies that

$$
\left\|f-S_{P}(f)\right\| \leq K\left(\sigma_{m}(f, \Psi)+\varepsilon\right) .
$$

Let

$$
G_{N}^{\tau}(f, \Psi)=\sum_{k \in Q} c_{k}(f, \Psi) \psi_{k}=S_{Q}(f)
$$

Then

$$
\left\|f-G_{N}^{\tau}(f, \Psi)\right\| \leq\left\|f-S_{P}(f)\right\|+\left\|S_{P}(f)-S_{Q}(f)\right\| .
$$

The first term on the right side of (3.3) has been estimated in (3.2). We now estimate the second term. We have

$$
S_{P}(f)-S_{Q}(f)=S_{P \backslash Q}(f)-S_{Q \backslash P}(f) .
$$

Similarly to (3.2) we have

$$
\left\|S_{Q \backslash P}(f)\right\| \leq K\left(\sigma_{m}(f, \Psi)+\varepsilon\right) .
$$

We now estimate $\left\|S_{P \backslash Q}(f)\right\|$. Let $J$ be the set of indices $i$ such that the elements of $P \cap Q$ were chosen at steps $i \in J$. Set

$$
a:=\max _{k \in P \backslash Q}\left|c_{k}(f, \Psi)\right| .
$$

Then from the definition of the WTGA we obtain

$$
S_{Q \backslash P}(f)=\sum_{k \in Q \backslash P} c_{k}(f, \Psi) \psi_{k}
$$

and $\left\{c_{k}(f, \Psi)\right\}_{k \in Q \backslash P}$ can be enumerated by indices $i \in V:=[1, N] \backslash J$ in such a way that

$$
\left|c_{k_{i}}(f, \Psi)\right| \geq t_{i} a, \quad i \in V .
$$

Then by Proposition 3.1 we have

$$
\left\|S_{Q \backslash P}(f)\right\| \geq K^{-1} a \phi(\tau, N, V)
$$

and

$$
\left\|S_{P \backslash Q}(f)\right\| \leq \operatorname{Ka\varphi }^{\mathrm{s}}(\#(P \backslash Q)) .
$$


Thus in the case of $N=m$ (Theorem 4) setting $n:=\#(P \backslash Q)=\#(Q \backslash P)$ we get

$$
\left\|S_{P \backslash Q}(f)\right\| \leq K^{2} \frac{\varphi^{\mathrm{s}}(n)}{\phi(\tau, m, n)}\left\|S_{Q \backslash P}(f)\right\| \leq K^{2} \mu(\tau, m)\left\|S_{Q \backslash P}(f)\right\| .
$$

In the case of $N=v_{m}$ (Theorem 5) we obtain

$$
\phi(\tau, N, V) \geq \phi(\tau, N)-\left\|\sum_{i \in J} t_{i} \psi_{k_{i}}\right\| \geq \phi(\tau, N)-\varphi(m) \geq \varphi(m) .
$$

Combining (3.6)-(3.8) we get

$$
\left\|S_{P \backslash Q}(f)\right\| \leq K^{2}\left\|S_{Q \backslash P}\right\| .
$$

It remains to substitute this inequality and (3.5) into (3.4) and use (3.3).

Theorems 4 and 5 are proved.

Let us make some comments on Theorems 4 and 5. First we consider the case when $\Psi$ is a greedy basis. Then by Definition 1.1 we have (1.3) satisfied. Let us see what Theorem 4 gives in this case. We recall a result from [KT].

Definition 3.1. We say that a normalized basis $\Psi=\left\{\psi_{k}\right\}_{k=1}^{\infty}$ is a democratic basis for $X$ if there exists a constant $D:=D(X, \Psi)$ such that for any two finite sets of indices $P$ and $Q$ with the same cardinality we have

$$
\left\|\sum_{k \in P} \psi_{k}\right\| \leq D\left\|\sum_{k \in Q} \psi_{k}\right\|
$$

The following theorem was proved in $[\mathrm{KT}]$.

TheOREM 3.1. A normalized basis is greedy if and only if it is unconditional and democratic.

Thus by Theorem 3.1 a greedy basis satisfies (3.9). It is easy to see that (3.9) implies $\varphi^{\mathrm{s}}(m) \leq D \phi(m)$ and therefore for $\tau=\{1\}$ we get $\mu(\{1\}, m) \leq D$. This means that Theorem 4 states that for any greedy basis $\Psi$ we have (1.3) for any $\varrho \in D(f)$.

We now apply Theorems 4 and 5 for $\Psi=\mathcal{H}_{p}^{d}, 1<p<\infty$, with the weakness sequence $\tau=\{1\}$. We will use the following known inequalities.

Lemma 3.1. Let $\Lambda$ be a set of indices with $\# \Lambda=m$. Then for $2 \leq p<\infty$,

$$
\begin{aligned}
C_{p, d}^{1} m^{1 / p} \min _{\mathbf{n} \in \Lambda}\left\|c_{\mathbf{n}} H_{\mathbf{n}}\right\|_{p} & \leq\left\|\sum_{\mathbf{n} \in \Lambda} c_{\mathbf{n}} H_{\mathbf{n}}\right\|_{p} \\
& \leq C_{p, d}^{2} m^{1 / p}(\log m)^{h(p, d)} \max _{\mathbf{n} \in \Lambda}\left\|c_{\mathbf{n}} H_{\mathbf{n}}\right\|_{p},
\end{aligned}
$$


and for $1<p \leq 2$,

$$
\begin{aligned}
C_{p, d}^{3} m^{1 / p}(\log m)^{-h(p, d)} \min _{\mathbf{n} \in \Lambda}\left\|c_{\mathbf{n}} H_{\mathbf{n}}\right\|_{p} & \leq\left\|\sum_{\mathbf{n} \in \Lambda} c_{\mathbf{n}} H_{\mathbf{n}}\right\|_{p} \\
& \leq C_{p, d}^{4} m^{1 / p} \max _{\mathbf{n} \in \Lambda}\left\|c_{\mathbf{n}} H_{\mathbf{n}}\right\|_{p},
\end{aligned}
$$

where $h(p, d):=(d-1)|1 / 2-1 / p|$.

Lemma 3.1 in the case $d=2,4 / 3 \leq p \leq 4$ has been proved in [T3] and in the general case in [W]. Lemma 3.1 implies that for $1<p<\infty$,

$$
\begin{aligned}
\mu(\{1\}, m) & \asymp C(p, d)(\log m)^{(d-1)|1 / 2-1 / p|}, \\
v_{m} & \asymp C(p, d) m(\log m)^{(d-1)|p / 2-1|} .
\end{aligned}
$$

Therefore Theorem 4 gives the known result (see [T3], [W])

$$
\begin{aligned}
\| f-G_{m} & \left(f, \mathcal{H}_{p}^{d}\right) \|_{p} \\
& \leq C(p, d)(\log m)^{(d-1)|1 / 2-1 / p|} \sigma_{m}\left(f, \mathcal{H}_{p}^{d}\right)_{p}, \quad 1<p<\infty .
\end{aligned}
$$

Theorem 5 gives a new result. We note that for functions $f$ with slow decay of $\sigma_{m}\left(f, \mathcal{H}_{p}^{d}\right)_{p}$ Theorem 5 gives a better estimate than (3.10). Consider for example $\sigma_{m}\left(f, \mathcal{H}_{p}^{d}\right)_{p} \asymp m^{-\alpha}$. Then (3.10) gives

$$
\left\|f-G_{m}\left(f, \mathcal{H}_{p}^{d}\right)\right\|_{p} \ll(\log m)^{(d-1)|1 / 2-1 / p|} m^{-\alpha}, \quad 1<p<\infty,
$$

while Theorem 5 gives

$$
\left\|f-G_{m}\left(f, \mathcal{H}_{p}^{d}\right)\right\|_{p} \ll\left(m(\log m)^{-(d-1)|p / 2-1|}\right)^{-\alpha}, \quad 1<p<\infty .
$$

For $\alpha<1 / p$ the estimate (3.12) is better than (3.11).

Let us now discuss optimality of the sequence $\left\{v_{m}\right\}$.

TheOrem 3.2. Let $X$ be a Banach space with a normalized unconditional basis $\Psi$. Let $\tau=\left\{t_{n}, n \geq 1\right\}$ be a weakness sequence such that the $W T G A$ with respect to $\Psi$ and $\tau$ is convergent. Let $\left\{v_{m}, m \in \mathbb{N}\right\}$ be a sequence of natural numbers with $v_{m} \geq m$. Then the following two conditions are equivalent.

(i) There is a constant $C$ such that for each pair of natural numbers $n \leq m$ and any set $V \subseteq\left[1, v_{m}\right]$ with $\# V=v_{m}-m+n$ we have

$$
\left\|\sum_{j \in A} \psi_{j}\right\| \leq C\left\|\sum_{i \in V} t_{i} \psi_{k_{i}}\right\|
$$

for any two sets of indices $A$ and $B:=\left\{k_{i}, i \in V\right\}\left(\right.$ all $k_{i}, i \in V$, are different) satisfying $A \cap B=\emptyset$ and $\# A=n$.

(ii) There is a $C>0$ such that for all $f \in X$ and $m \in \mathbb{N}$,

$$
\left\|f-G_{v_{m}}^{\tau}(f, \Psi)\right\| \leq C \sigma_{m}(f, \Psi) .
$$


Proof. The implication (i) $\Rightarrow$ (ii) can be proved in the same way as Theorem 5 . We will not dwell on it here. We only note that we use (i) with $A=P \backslash Q$ and $B=Q \backslash P$ to get from the following analogs of (3.6) and (3.7):

$$
\begin{gathered}
\left\|S_{Q \backslash P}(f)\right\| \geq K^{-1} a\left\|\sum_{i \in V} t_{i} \psi_{k_{i}}\right\|, \quad B=\left\{k_{i}, i \in V\right\}, \\
\left\|S_{P \backslash Q}(f)\right\| \leq K a\left\|\sum_{n \in A} \psi_{n}\right\|,
\end{gathered}
$$

the inequality

$$
\left\|S_{P \backslash Q}(f)\right\| \leq C K^{2}\left\|S_{Q \backslash P}(f)\right\| .
$$

We now prove that (ii) $\Rightarrow$ (i). Let $n \leq m$ be given and let $V, A, B$ satisfy the conditions of (i). Let $Y$ be such that $\# Y=m-n$ and $A \cap Y=\emptyset$ and $B \cap Y=\emptyset$. Consider

$$
f:=\sum_{n \in A \cup Y} \psi_{n}+\sum_{i \in V} t_{i} \psi_{k_{i}} .
$$

We take the following realization of the WTGA. For steps $i \in V$ we take $n_{i}=k_{i}$ and for steps $i \notin V$ we take different $n_{i} \in Y$. Then we get

$$
G_{v_{m}}^{\tau}(f, \Psi)=\sum_{n \in Y} \psi_{n}+\sum_{i \in V} t_{i} \psi_{k_{i}}
$$

This implies by (ii) that

$$
\left\|\sum_{n \in A} \psi_{n}\right\|=\left\|f-G_{v_{m}}^{\tau}(f, \Psi)\right\| \leq C \sigma_{m}(f, \Psi) \leq C\left\|\sum_{i \in V} t_{i} \psi_{k_{i}}\right\| .
$$

This completes the proof of Theorem 3.2.

Let us make some more comments on Theorems 4 and 5 .

It is well known that for $\tau=\{1\}$ and a normalized unconditional basis $\Psi$, the extra factor $\mu(\{1\}, m)$ in Theorem 4 is finite and $\mu(\{1\}, m) \leq C m$ (see e.g. Corollaries to Theorem 5 in $[\mathrm{W}]$ or formula (1.8) in [Os]). However, even for $\tau=\{1\}$ it may happen that there is no sequence $\left\{v_{m}\right\}$ such that inequality (3.13) holds for all $f \in X$ and $m$, with a constant $C$ independent of $f$ and $m$. A simple example of such a space $X$ and an unconditional basis $\Psi$ is $X=l_{1} \oplus c_{0}$ with $\Psi=\left\{e_{n}, n \in \mathbb{N}\right\} \cup\left\{f_{n}, n \in \mathbb{N}\right\},\left\{e_{n}, n \in \mathbb{N}\right\}$ and $\left\{f_{n}, n \in \mathbb{N}\right\}$ being the unit vector bases in $l_{1}$ and $c_{0}$, respectively: for any finite $A \subset \mathbb{N}$ one has $\left\|\sum_{n \in A} e_{n}\right\|=\# A$ and $\left\|\sum_{n \in A} f_{n}\right\|=1$, which means that condition (i) of Theorem 3.2 cannot be satisfied by any sequence $\left\{v_{m}\right\}$. This example has been suggested to us by the referee and replaces our previous, more complicated example. 
4. Greedy subsequences of the multivariate Haar basis. It is well known that for $p \neq 2$ and $d \geq 2$ the $d$-variate tensor product Haar system $\mathcal{H}_{p}^{d}$ is not a greedy basis in the corresponding $L_{p}\left([0,1]^{d}\right)$ space. However, for some functions the Thresholding Greedy Algorithm may give an order of approximation comparable with the order of best approximation. In this section we address the question: For what functions does the TGA realize nearly best $m$-term approximation? Let us recall that for $\mathbf{s}=\left(s_{1}, \ldots, s_{d}\right)$, the dyadic block $U_{\mathbf{s}}$ is defined by (1.10), and the Haar functions $H_{\mathbf{n}, p}$ with $\mathbf{n} \in U_{\mathbf{s}}$ have the same shape of supports.

We are interested in the influence of some "structural constraints" imposed on a function on the efficiency of TGA with respect to the Haar system $\mathcal{H}_{p}^{d}$. By "structural constraints" we mean constraints imposed on the number of nonzero coefficients in dyadic blocks or on the number of dyadic blocks with nonzero coefficients. These constraints are expressed in terms of classes of sequences $\mathbf{R}(K), \mathbf{J}(K)$, and $\mathbf{G}(d)$ (see the Introduction). We begin by proving Theorem 6 .

Proof of Theorem 6. For any sequence $\mathcal{M}$ the system $\mathcal{H}_{p}^{d}[\mathcal{M}]$ is an unconditional basis for $L_{p}[\mathcal{M}], 1<p<\infty$. Thus by Theorem 3.1 it is sufficient to establish that $\mathcal{H}_{p}^{d}[\mathcal{M}]$ is democratic provided $\mathcal{M} \in \mathbf{G}(d)$. This follows from Lemmas 4.1 and 4.2 below.

Lemma 4.1. Let $1<p<\infty$ and $\mathcal{M} \in \mathbf{R}(K)$. Then for any different $\mathbf{n}_{1}, \ldots, \mathbf{n}_{m} \in \mathcal{M}$ we have

$$
\left\|\sum_{k=1}^{m} H_{\mathbf{n}_{k}, p}\right\|_{p} \asymp m^{1 / p}
$$

with constants depending only on $K, d$ and $p$.

Lemma 4.2. Let $1<p<\infty$ and $\mathcal{M} \in \mathbf{J}(K)$. Then for any different $\mathbf{n}_{1}, \ldots, \mathbf{n}_{m} \in \mathcal{M}$ we have

$$
\left\|\sum_{k=1}^{m} H_{\mathbf{n}_{k}, p}\right\|_{p} \asymp m^{1 / p}
$$

with constants depending only on $K, d$ and $p$.

For $d=1$ Lemma 4.1 with $\mathcal{M}=\mathbb{N}$ was proved in [T2]. That same proof works for $d \geq 2$ under the assumption $\mathcal{M} \in \mathbf{R}(K)$ (see also the proof of Lemma 9 in $[\mathrm{W}])$. Let us prove Lemma 4.2 .

Proof of Lemma 4.2. We recall (see Lemma 3.1) that by the LittlewoodPaley theory we have

$$
\left\|\sum_{k=1}^{m} H_{\mathbf{n}_{k}, p}\right\|_{p} \leq C(p, d) m^{1 / p} \quad \text { for } 1<p \leq 2
$$


and

$$
C(p, d) m^{1 / p} \leq\left\|\sum_{k=1}^{m} H_{\mathbf{n}_{k}, p}\right\|_{p} \quad \text { for } 2 \leq p<\infty
$$

for any different $\mathbf{n}_{1}, \ldots, \mathbf{n}_{m}$. To prove the upper estimate in the case $2<$ $p<\infty$, we use the following inequality, which is a special case of Lemma 2.3 of [T1]: for $2<p<\infty$ and $f=\sum_{\mathbf{s}} f_{\mathbf{s}}$ with $f_{\mathbf{s}}=\sum_{\mathbf{n} \in U_{\mathbf{s}}} c_{\mathbf{n}}(f) H_{\mathbf{n}}$,

$$
\|f\|_{p} \leq C_{p, d}\left(\sum_{\mathbf{s}}\left(2^{|\mathbf{s}|(1 / 2-1 / p)}\left\|f_{\mathbf{s}}\right\|_{2}\right)^{p}\right)^{1 / p} .
$$

For each $\mathbf{s}$, let $m_{\mathbf{s}}$ be the number of $\mathbf{n}_{k}$ 's in $U_{\mathbf{s}}$. Note that

$$
\left\|\sum_{k: \mathbf{n}_{k} \in U_{\mathbf{s}}} H_{\mathbf{n}_{k}, p}\right\|_{2}=2^{|\mathbf{s}|(1 / p-1 / 2)} m_{\mathbf{s}}^{1 / 2}
$$

and therefore by (4.1),

$$
\left\|\sum_{k=1}^{m} H_{\mathbf{n}_{k}, p}\right\|_{p} \leq C_{p, d}\left(\sum_{\mathbf{s}} m_{\mathbf{s}}^{p / 2}\right)^{1 / p} .
$$

Taking into account that $m=\sum_{\mathbf{s}} m_{\mathbf{s}}$ and $m_{\mathbf{s}} \leq K$ by the assumption $\mathcal{M} \in \mathbf{J}(K)$ we get

$$
\left\|\sum_{k=1}^{m} H_{\mathbf{n}_{k}, p}\right\|_{p} \leq C_{p, d} m^{1 / p} \quad \text { for } 2<p<\infty
$$

with the constant depending only on $p, d$ and $K$.

To complete the proof, recall that the lower estimate in the case $1<p<2$ follows from the upper estimates for all $2<p<\infty$ by duality. Using the Hölder inequality we obtain

$$
\begin{aligned}
m & =\sum_{k=1}^{m} \int_{[0,1]^{d}} H_{\mathbf{n}_{k}, p}(x) \cdot H_{\mathbf{n}_{k}, p^{\prime}}(x) d x \\
& =\int_{[0,1]^{d}}\left(\sum_{k=1}^{m} H_{\mathbf{n}_{k}, p}(x)\right) \cdot\left(\sum_{k=1}^{m} H_{\mathbf{n}_{k}, p^{\prime}}(x)\right) d x \\
& \leq\left\|\sum_{k=1}^{m} H_{\mathbf{n}_{k}, p}\right\|_{p} \cdot\left\|\sum_{k=1}^{m} H_{\mathbf{n}_{k}, p^{\prime}}\right\|_{p^{\prime}} \leq C m^{1 / p^{\prime}}\left\|\sum_{k=1}^{m} H_{\mathbf{n}_{k}, p}\right\|_{p},
\end{aligned}
$$

which gives the lower estimate in the case $1<p<2$ with a constant depending only on $p, d$ and $K$.

This completes the proof of Theorem 6 .

We now proceed to a discussion of in what sense Theorem 6 is sharp. We need some more notation describing the structural constraints on functions. 
Let $\Lambda=\left\{\lambda_{\mathbf{s}}, \mathbf{s}=\left(s_{1}, \ldots, s_{d}\right) \in \mathbb{Z}_{+}^{d}\right\}$ be a sequence of integers satisfying

$$
0 \leq \lambda_{\mathbf{s}} \leq \# U_{\mathbf{s}}
$$

Define

$$
\mathcal{V}(\Lambda):=\left\{\mathcal{M}: \#\left(\mathcal{M} \cap U_{\mathbf{s}}\right) \leq \lambda_{\mathbf{s}}\right\}
$$

For $1<p<\infty$ consider the following sets of functions:

$$
L_{p}(\Lambda)=\bigcup_{\mathcal{M} \in \mathcal{V}(\Lambda)} L_{p}[\mathcal{M}]
$$

i.e. $L_{p}(\Lambda)$ consists of $f \in L_{p}\left([0,1]^{d}\right)$ with at most $\lambda_{\mathbf{s}}$ nonzero coefficients in blocks $U_{\mathbf{s}}, \mathbf{s} \in \mathbb{Z}_{+}^{d}$.

We describe a distribution of $\lambda_{\mathbf{s}}$ 's for a given sequence $\Lambda$ by defining, for nonnegative integers $\mu, M$,

$$
\alpha_{\mu, M}(\Lambda):=\#\left\{\mathbf{s}:|\mathbf{s}|=\mu \text { and } \lambda_{\mathbf{s}} \geq M\right\} .
$$

Now, let $A:=\left\{a_{\mu, M}\right\}$ be a sequence of nonnegative integers satisfying

$$
\begin{gathered}
a_{\mu, M_{1}} \leq a_{\mu, M_{2}} \quad \text { for } M_{1} \geq M_{2} \\
a_{\mu, 0}=\#\left\{\mathbf{s}=\left(s_{1}, \ldots, s_{d}\right):|\mathbf{s}|=\mu\right\}, \quad a_{\mu, M}=0 \quad \text { for } M>\max _{|\mathbf{s}|=\mu} \# U_{\mathbf{s}} .
\end{gathered}
$$

Note that the sequence $\left\{\alpha_{\mu, M}(\Lambda)\right\}$ defined above satisfies these conditions for any $\Lambda$.

To formulate the next result, we define a type of a sequence $\Lambda$ and full range sequences.

Definition 4.1. Let $A=\left\{a_{\mu, M}\right\}$ be a sequence satisfying (4.5) and (4.6), and let $\Lambda=\left\{\lambda_{\mathbf{s}}\right\}$ be a sequence of integers satisfying (4.2). $\Lambda$ is called a type $A$ sequence if $\alpha_{\mu, M}(\Lambda)=a_{\mu, M}$ for all $\mu, M \geq 0$ (where $\alpha_{\mu, M}(\Lambda)$ is given by (4.4)).

Definition 4.2. Let $A=\left\{a_{\mu, M}\right\}$ be a sequence satisfying (4.5) and (4.6). It is called a full range sequence if $\limsup _{\mu \rightarrow \infty} a_{\mu, M}=\infty$ for each $M>0$.

Take a sequence $\mathcal{M} \in \mathbf{G}(d)$ and define

$$
a_{\mu, M}(\mathcal{M}):=\#\left\{\mathbf{s}:|\mathbf{s}|=\mu \text { and } \#\left(\mathcal{M} \cap U_{\mathbf{s}}\right) \geq M\right\} .
$$

From the definition of $\mathbf{G}(d)$ we get

$$
\mathcal{M}=\mathcal{M}_{1} \cup \mathcal{M}_{2}, \quad \mathcal{M}_{1} \in \mathbf{R}\left(K_{1}\right), \quad \mathcal{M}_{2} \in \mathbf{J}\left(K_{2}\right) .
$$

Thus for $M>K_{2}$ we have $a_{\mu, M}(\mathcal{M}) \leq K_{1}$. Therefore any $\mathcal{M} \in \mathbf{G}(d)$ has a distribution that is not a full range sequence. It follows from Definition 4.2 that the opposite is also true: if $\left\{a_{\mu, M}(\mathcal{M})\right\}$ is not a full range sequence then $\mathcal{M} \in \mathbf{G}(d)$. Theorem 4.1 below states that if constraints on the structure of 
a function are given in terms of the distribution sequence $\left\{a_{\mu, M}(\mathcal{M})\right\}$ then Theorem 6 is the best possible.

Theorem 4.1. Let $A=\left\{a_{\mu, M}\right\}$ be a sequence satisfying (4.5) and (4.6). Let $d \geq 2,1<p<\infty, p \neq 2$. Then the following conditions are equivalent:

(i) $A$ is not a full range sequence.

(ii) There is a constant $C=C(A, d, p)$ (depending only on $A, d$ and $p$ ) such that for each $\Lambda=\left\{\lambda_{\mathbf{s}}, \mathbf{s}=\left(s_{1}, \ldots, s_{d}\right)\right\}$ of type $A$ we have, for all $f \in L_{p}(\Lambda)$ and $m \in \mathbb{N}$,

$$
\left\|f-G_{m}\left(f, \mathcal{H}_{p}^{d}\right)\right\|_{p} \leq C \sigma_{m}\left(f, \mathcal{H}_{p}^{d}\right) .
$$

Proof. If $A$ is not a full range sequence, then for some $M, L$ we have $a_{\mu, M} \leq L$ for all $\mu \geq 0$, and each $\mathcal{M}$ satisfying $a_{\mu, M}(\mathcal{M}) \leq a_{\mu, M}$ can be written as $\mathcal{M}=\mathcal{M}_{1} \cup \mathcal{M}_{2}$ with $\mathcal{M}_{1} \in \mathbf{R}(L)$ and $\mathcal{M}_{2} \in \mathbf{J}(M)$. The implication (i) $\Rightarrow$ (ii) now follows from Lemmas 4.1 and 4.2 .

We now prove that (ii) $\Rightarrow$ (i). For any given sequence $A$ of full range we will construct a $\Lambda$ of type $A$ such that (4.7) does not hold. We begin with a construction which will provide us with building blocks of the counterexample sequence $\mathcal{M}$. This construction is a modification of a construction from [T3, Section 4].

For a given pair of natural numbers $k$ and $l$ such that $l<k$ we consider the following special polynomials. First, set

$$
I(k, l):=\left\{\mathbf{s}:|\mathbf{s}|=k d, s_{j} \geq k-l, j=1, \ldots, d\right\} .
$$

Then

$$
\# I(k, l) \asymp l^{d-1} .
$$

Consider the cube $\left[0,2^{l-k}\right)^{d}$ and define

$$
\begin{aligned}
U_{\mathbf{s}}(k, l) & :=\left\{\mathbf{n}: \mathbf{n} \in U_{\mathbf{s}} \text { and } \operatorname{supp} H_{\mathbf{n}} \subseteq\left[0,2^{l-k}\right)^{d}\right\}, \\
E(k, l) & :=\bigcup_{\mathbf{s} \in I(k, l)} U_{\mathbf{s}}(k, l) .
\end{aligned}
$$

Define a polynomial

$$
g_{k, l}:=\sum_{\mathbf{n} \in E(k, l)} H_{\mathbf{n}, p} .
$$

By the Littlewood-Paley theory we have

$$
\left\|g_{k, l}\right\|_{p} \asymp\left\|\left(\sum_{\mathbf{n} \in E(k, l)}\left|H_{\mathbf{n}, p}(x)\right|^{2}\right)^{1 / 2}\right\|_{p}, \quad 1<p<\infty .
$$

The supports of $\sum_{\mathbf{n} \in U_{\mathbf{s}}(k, l)} H_{\mathbf{n}, p}, \mathbf{s} \in I(k, l)$, cover the cube $\left[0,2^{l-k}\right)^{d}$ and therefore from (4.8) we obtain

$$
\left\|g_{k, l}\right\|_{p} \asymp 2^{l d / p} l^{(d-1) / 2} .
$$


The number $m:=m(k, l)$ of terms of the polynomial $g_{k, l}$ satisfies

$$
C_{1}(d) l^{d-1} 2^{l d} \leq m \leq C_{2}(d) l^{d-1} 2^{l d} .
$$

Take a companion (to $g_{k, l}$ ) polynomial

$$
h_{m}:=\sum_{i=1}^{m} H_{\mathbf{n}_{i}, p}
$$

such that $\mathbf{n}_{i} \notin E(k, l)$ for $i=1, \ldots, m$ and

$$
\operatorname{supp} H_{\mathbf{n}_{i}} \cap \operatorname{supp} H_{\mathbf{n}_{j}}=\emptyset, \quad i \neq j .
$$

Then

$$
\left\|h_{m}\right\|_{p} \asymp m^{1 / p} .
$$

Considering the function $f:=g_{k, l}+2 h_{m}$ in the case $2<p<\infty$ and $f:=2 g_{k, l}+h_{m}$ in the case $1<p<2$ we will get, for an $\mathcal{M}$ containing $E(k, l)$ and $\left\{\mathbf{n}_{i}\right\}_{i=1}^{m}$,

$$
\left\|f-G_{m}\left(f, \mathcal{H}_{p}^{d}[\mathcal{M}]\right)\right\|_{p} / \sigma_{m}\left(f, \mathcal{H}_{p}^{d}[\mathcal{M}]\right)_{p} \gg(\log m)^{(d-1)|1 / p-1 / 2|} .
$$

Let $A$ be a full range sequence. Then there is an increasing sequence $\left\{\mu_{l}\right\}$ such that

$$
a_{d \mu_{l}, 2^{l d}} \geq C l^{d-1}+1
$$

where $C$ is such that $\# I(k, l) \leq C l^{d-1}$ for all $k, l$. We define

$$
\mathcal{M}:=\left(\bigcup_{l=1}^{\infty} E\left(\mu_{l}, l\right)\right) \cup\left(\left\{\mathbf{n}_{j}\right\}_{j=1}^{\infty}\right),
$$

where $\left\{\mathbf{n}_{j}\right\}_{j=1}^{\infty}$ is such that

$$
\operatorname{supp} H_{\mathbf{n}_{j}} \subset[1 / 2,1)^{d}, \quad \operatorname{supp} H_{\mathbf{n}_{i}} \cap \operatorname{supp} H_{\mathbf{n}_{j}}=\emptyset, \quad i \neq j .
$$

It is clear that $\left\{\mathbf{n}_{j}\right\}_{j=1}^{\infty}$ with the properties (4.13) can be chosen in such a way that $a_{\mu, M}(\mathcal{M}) \leq a_{\mu, M}$ for all $\mu, M$. To complete the proof of Theorem 4.1 , it is enough to take any $\Lambda$ of type $A$ such that $\mathcal{M} \in \mathcal{V}(\Lambda)$.

We note that the above argument implies even more.

Proposition 4.1. Let $A=\left\{a_{\mu, M}\right\}$ be a full range sequence and $d \geq 2$, $1<p<\infty$. Let $\{C(m, A, d, p), m \in \mathbb{N}\}$ be a sequence of reals such that for each $\Lambda=\left\{\lambda_{\mathbf{s}}\right\}$ of type $A, f \in L_{p}(\Lambda)$ and $m \in \mathbb{N}$,

$$
\left\|f-G_{m}\left(f, \mathcal{H}_{p}^{d}\right)\right\|_{p} \leq C(m, A, p) \sigma_{m}\left(f, \mathcal{H}_{p}^{d}\right)_{p}
$$

Then

$$
C(m, A, d, p) \asymp(\log m)^{(d-1)|1 / 2-1 / p|} .
$$

5. Some direct and inverse theorems in $m$-term approximation with regard to $\mathcal{H}_{p}^{d}$. In the case $d=1$ the Haar basis is a greedy basis for 
$L_{p}, 1<p<\infty$. The following characterization theorem has been established in [T3] (for the case $p=2$ see [St], [DT]). We will use the notation

$$
a_{n}(f, p):=\left|c_{k_{n}}\left(f, \mathcal{H}_{p}^{d}\right)\right|
$$

for the decreasing rearrangement of the coefficients of $f$.

Theorem 5.1. Let $d=1,1<p<\infty$ and $0<q<\infty$. Then for any positive $r$ we have the equivalence

$$
\sum_{m=1}^{\infty} \sigma_{m}(f, \mathcal{H})_{p}^{q} m^{r q-1}<\infty \Leftrightarrow \sum_{n=1}^{\infty} a_{n}(f, p)^{q} n^{r q-1+q / p}<\infty .
$$

Let us recall the definition of the Lorentz spaces of sequences and introduce new spaces which provide a finer (logarithmic) scale. For a sequence $\left\{x_{k}\right\}_{k=1}^{\infty}$ let $\left\{x_{\varrho(k)}\right\}_{k=1}^{\infty}$ be a decreasing rearrangement

$$
\left|x_{\varrho(1)}\right| \geq\left|x_{\varrho(2)}\right| \geq \ldots
$$

For $r>0$ and $0<q<\infty$ let

$$
\ell_{q}^{r}:=\left\{\left\{x_{k}\right\}_{k=1}^{\infty}: \sum_{k=1}^{\infty}\left|x_{\varrho(k)}\right|^{q} k^{r q-1}<\infty\right\}
$$

or, equivalently,

$$
\ell_{q}^{r}:=\left\{\left\{x_{k}\right\}_{k=1}^{\infty}: \sum_{s=0}^{\infty}\left|x_{\varrho\left(2^{s}\right)}\right|^{q} 2^{r q s}<\infty\right\} .
$$

For $r>0, b \in \mathbb{R}$ and $0<q<\infty$ let

$$
\ell_{q}^{r, b}:=\left\{\left\{x_{k}\right\}_{k=1}^{\infty}: \sum_{s=1}^{\infty}\left(\left|x_{\varrho\left(2^{s}\right)}\right| 2^{r s} s^{b}\right)^{q}<\infty\right\} .
$$

It is clear that $\ell_{q}^{r, 0}=\ell_{q}^{r}$.

The proof of Theorem 5.1 was based on the following two lemmas.

Lemma 5.1. For any two positive integers $N<M$ we have

$$
a_{M}(f, p) \leq C(p) \sigma_{N}(f, \mathcal{H})_{p}(M-N)^{-1 / p} .
$$

LEMMA 5.2. For any sequence $m_{0}<m_{1}<\ldots$ of nonnegative integers we have

$$
\sigma_{m_{s}}(f, \mathcal{H})_{p} \leq C(p) \sum_{i=s}^{\infty} a_{m_{i}}(f, p)\left(m_{i+1}-m_{i}\right)^{1 / p} .
$$

We will prove in this section the following multivariate analogs of the above lemmas. 
Lemma 5.3. For any two positive integers $N<M$ we have

$$
\begin{array}{ll}
a_{M}(f, p) \leq C(p, d) \sigma_{N}\left(f, \mathcal{H}^{d}\right)_{p}(M-N)^{-1 / p}, & 2 \leq p<\infty, \\
a_{M}(f, p) \leq C(p, d) \sigma_{N}\left(f, \mathcal{H}^{d}\right)_{p}(M-N)^{-1 / p}(\log M)^{h(p, d)}, & 1<p \leq 2,
\end{array}
$$

with $h(p, d):=(d-1)|1 / 2-1 / p|$.

Lemma 5.4. For any sequence $m_{0}<m_{1}<\ldots$ of nonnegative integers we have

$$
\begin{array}{cc}
\sigma_{m_{s}}\left(f, \mathcal{H}^{d}\right)_{p} \leq C(p, d) \sum_{i=s}^{\infty} a_{m_{i}}(f, p)\left(m_{i+1}-m_{i}\right)^{1 / p}\left(\log m_{i+1}\right)^{h(p, d)}, & 2 \leq p<\infty \\
\sigma_{m_{s}}\left(f, \mathcal{H}^{d}\right)_{p} \leq C(p, d) \sum_{i=s}^{\infty} a_{m_{i}}(f, p)\left(m_{i+1}-m_{i}\right)^{1 / p}, & 1<p \leq 2 .
\end{array}
$$

Proof of Lemmas 5.3 and 5.4. Lemma 5.4 follows directly from Lemma 3.1. To prove Lemma 5.3, for given $f=\sum_{\mathbf{n}} c_{\mathbf{n}} H_{\mathbf{n}, p}$, let $\Lambda_{N}$ and $\left\{u_{\mathbf{n}}, \mathbf{n} \in \Lambda_{N}\right\}$ be the set of indices with $\# \Lambda_{N}=N$ and coefficients such that

$$
\left\|f-\sum_{\mathbf{n} \in \Lambda_{N}} u_{\mathbf{n}} H_{\mathbf{n}, p}\right\|_{p} \leq 2 \sigma_{N}\left(f, \mathcal{H}^{d}\right)_{p} .
$$

Moreover, let $G_{M}=\left\{\mathbf{n}_{1}, \ldots, \mathbf{n}_{M}\right\}$, where $a_{k}(f, p)=\left|c_{\mathbf{n}_{k}}\right|$. By unconditionality of $\mathcal{H}_{p}^{d}$ we have

$$
\begin{aligned}
\left\|f-\sum_{\mathbf{n} \in \Lambda_{N}} c_{\mathbf{n}} H_{\mathbf{n}, p}\right\|_{p} & \leq C\left\|f-\sum_{\mathbf{n} \in \Lambda_{N}} u_{\mathbf{n}} H_{\mathbf{n}, p}\right\|_{p} \leq 2 C \sigma_{N}\left(f, \mathcal{H}^{d}\right)_{p}, \\
a_{M}(f, p)\left\|\sum_{\mathbf{n} \in G_{M} \backslash \Lambda_{N}} H_{\mathbf{n}, p}\right\|_{p} & \leq C\left\|\sum_{\mathbf{n} \in G_{M} \backslash \Lambda_{N}} c_{\mathbf{n}} H_{\mathbf{n}, p}\right\|_{p} \\
& \leq C\left\|f-\sum_{\mathbf{n} \in \Lambda_{N}} c_{\mathbf{n}} H_{\mathbf{n}, p}\right\|_{p} .
\end{aligned}
$$

As \# $\left(G_{M} \backslash \Lambda_{N}\right) \geq M-N$, Lemma 5.3 now follows from Lemma 3.1.

Using Lemmas 5.3 and 5.4 one can establish the following embedding theorem in the same way as Theorem 5.1 was deduced from Lemmas 5.1 and 5.2 in [T3].

Theorem 5.2. Let $1<p<\infty$. Define

$$
\sigma(f)_{p}:=\left\{\sigma_{m}\left(f, \mathcal{H}^{d}\right)_{p}\right\}_{m=1}^{\infty}, \quad a(f, p):=\left\{a_{n}(f, p)\right\}_{n=1}^{\infty} .
$$

Then we have the implications:

$$
\begin{array}{ll}
\sigma(f)_{p} \in \ell_{q}^{r, b} \Rightarrow a(f, p) \in \ell_{q}^{r+1 / p, b}, & 2 \leq p<\infty, \\
\sigma(f)_{p} \in \ell_{q}^{r, b} \Rightarrow a(f, p) \in \ell_{q}^{r+1 / p, b-h(p, d)}, & 1<p \leq 2,
\end{array}
$$




$$
\begin{array}{ll}
a(f, p) \in \ell_{q}^{r+1 / p, b} \Rightarrow \sigma(f)_{p} \in \ell_{q}^{r, b-h(p, d)}, & 2 \leq p<\infty, \\
a(f, p) \in \ell_{q}^{r+1 / p, b} \Rightarrow \sigma(f)_{p} \in \ell_{q}^{r, b}, & 1<p \leq 2 .
\end{array}
$$

Let us discuss in more detail the implication (5.1). We want to understand what smoothness classes are natural for $m$-term approximation with regard to the basis $\mathcal{H}^{d}$ which is the tensor product of the univariate Haar basis $\mathcal{H}$. We consider the relation $a(f, p) \in \ell_{q}^{r+1 / p, b}$ for a special choice of $b=0$ and $q=\xi:=(r+1 / p)^{-1}$. Then $a(f, p) \in \ell_{q}^{r+1 / p}$ is equivalent to $\sum_{n} a_{n}(f, p)^{\xi}<\infty$ or

$$
\sum_{\mathbf{n}}\left\|c_{\mathbf{n}}(f) H_{\mathbf{n}}\right\|_{p}^{\xi}<\infty, \quad \text { where } \quad f=\sum_{\mathbf{n}} c_{\mathbf{n}}(f) H_{\mathbf{n}} .
$$

Next, for $\mathbf{n} \in U_{\mathbf{s}}$ we have

$$
\left\|c_{\mathbf{n}}(f) H_{\mathbf{n}}\right\|_{p}=\left\|c_{\mathbf{n}}(f) H_{\mathbf{n}}\right\|_{\xi} 2^{-|\mathbf{s}|(1 / p-1 / \xi)}=\left\|c_{\mathbf{n}}(f) H_{\mathbf{n}}\right\|_{\xi} 2^{-r|\mathbf{s}|} .
$$

Thus (5.5) is equivalent to

$$
\sum_{\mathbf{s}}\left(2^{-r|\mathbf{s}|} \sum_{\mathbf{n} \in U_{\mathbf{s}}}\left\|c_{\mathbf{n}}(f) H_{\mathbf{n}}\right\|_{\xi}\right)^{\xi}<\infty .
$$

The above relation says that $f$ belongs to the mixed smoothness Besov class $M B_{\xi}^{r}\left(L_{\xi}\right)$. Thus we conclude that the multivariate classes with mixed smoothness are natural for studying nonlinear $m$-term approximation with regard to a basis which is a tensor product of univariate bases. There is an extensive literature on approximation theory in function classes with mixed smoothness. For the linear theory see [Te1], [Te2] and for some results on nonlinear $m$-term approximation see [T4] and [T6].

\section{References}

[CF] A. Córdoba and P. Fernández, Convergence and divergence of decreasing rearranged Fourier series, SIAM J. Math. Anal. 29 (1998), 1129-1139.

[D] R. A. DeVore, Nonlinear approximation, Acta Numerica 1998, 51-150.

[DJP] R. DeVore, B. Jawerth, and V. Popov, Compression of wavelet decompositions, Amer. J. Math. 114 (1992), 737-785.

[DKK] S. J. Dilworth, N. J. Kalton, and D. Kutzarova, On the existence of almost greedy bases in Banach spaces, Studia Math. 159 (2003), 67-101.

[DKKT] S. J. Dilworth, N. J. Kalton, D. Kutzarova, and V. N. Temlyakov, The tresholding greedy algorithm, greedy bases, and duality, Constr. Approx. 19 (2003), 575-597.

[DM] S. J. Dilworth and D. Mitra, A conditional quasi-greedy basis of $L_{1}$, Studia Math. 144 (2001), 95-100.

[DT] R. A. DeVore and V. N. Temlyakov, Some remarks on greedy algorithms, Adv. Comput. Math. 5 (1996), 173-187. 
[KP] M. I. Kadec and A. Pełczyński, Bases, lacunary sequences, and complemented subspaces in the spaces $L_{p}$, Studia Math. 21 (1962), 161-176.

[KT] S. V. Konyagin and V. N. Temlyakov, A remark on greedy approximation in Banach spaces, East J. Approx. 5 (1999), 1-15.

[KTe] - - - Greedy approximation with regard to bases and general minimal systems, Serdica Math. J. 28 (2002), 305-328.

[LT] J. Lindenstrauss and L. Tzafriri, Classical Banach Spaces I, II, Springer, Berlin, 1979.

[LTe] E. D. Livshits and V. N. Temlyakov, On convergence of Weak Greedy Algorithms, Tr. Mat. Inst. Steklova 232 (2001), 236-247 (in Russian); transl.: Proc. Steklov Inst. Math. 232 (2001), 229-239.

[Os] P. Oswald, Greedy algorithms and best $m$-term approximation with respect to biorthogonal systems, J. Fourier Anal. Appl. 7 (2001), 325-341.

[St] S. B. Stechkin, On absolute convergence of orthogonal series, Dokl. Akad. Nauk SSSR 102 (1955), 37-40 (in Russian).

[T1] V. N. Temlyakov, Some inequalities for multivariate Haar polynomials, East J. Approx. 1 (1995), 61-72.

[T2] - , The best m-term approximation and greedy algorithms, Adv. Comput. Math. 8 (1998), 249-265.

[T3] - Nonlinear $m$-term approximation with regard to the multivariate Haar system, East J. Approx. 4 (1998), 87-106.

[T4] - Greedy algorithms with regard to multivariate systems with special structure, Constr. Approx. 16 (2000), 399-425.

[T5] - Greedy algorithm and m-term trigonometric approximation, ibid. 14 (1998), $569-587$.

[T6] - Universal bases and greedy algorithms, ibid. 18 (2002), 529-550.

[T7] -, Weak greedy algorithms, Adv. Comput. Math. 12 (2000), 213-227.

[T8] - Nonlinear approximation with regard to bases, in: Approximation Theory X (St. Louis, MO, 2001), Vanderbilt Univ. Press, Nashville, TN, 2002, 373-402.

[T9] -, A criterion for convergence of weak greedy algorithms, Adv. Comput. Math. 17 (2002), 269-280.

[Te1] -, Approximation of functions with bounded mixed derivative, Proc. Steklov Institute (1989, Issue 1).

[Te2] -, Approximation of Periodic Functions, Nova Sci. Publ., New York, 1993.

[W] P. Wojtaszczyk, Greedy algorithms for general systems, J. Approx. Theory 107 (2000), 293-314.

[Wo] - Greedy type bases in Banach spaces, in: Constructive Theory of Functions (Varna, 2002), B. Bojanov (ed.), DARBA, Sofia, 2003, 136-155.

Institute of Mathematics

Polish Academy of Sciences

Abrahama 18

81-825 Sopot, Poland

E-mail: A.Kamont@impan.gda.pl
Department of Mathematics University of South Carolina Columbia, SC 29208, U.S.A. E-mail: temlyak@math.sc.edu

Received December 19, 2002

Revised version May 21, 2003 